\title{
Quantitative Clinical Rheumatology: "Keep It Simple, Stupid": MDHAQ Function, Pain, Global, and RAPID3 Quantitative Scores to Improve and Document the Quality of Rheumatologic Care
}

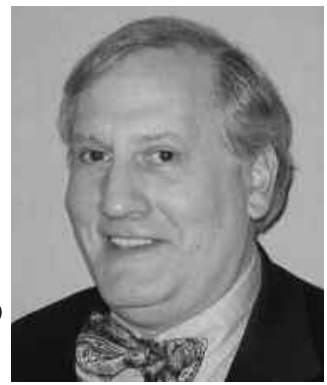
Q

"The KISS principle (acronym for "Keep It Simple, Stupid") states that design simplicity should be a key goal and unnecessary complexity avoided.... Extra features are not needed; an approach that seems "too easy to be true" is in fact the best way."

— Wikipedia (http://en.wikipedia.org)

The KISS principle ("Keep It Simple, Stupid") has been applied effectively in many disciplines, including "software development, animation, photography, engineering, and strategic planning" (Wikipedia). In clinical medicine, perhaps the most elegant example of the KISS principle involves simple laboratory tests such as hemoglobin, creatinine, etc., that may be applied to diagnosis, prognosis, management, and documentation of outcomes of diseases.

The discovery of rheumatoid factor $(\mathrm{RF})^{1}$ and antinuclear antibodies (ANA) ${ }^{2}$ in 1949 raised hope that similar, simple biomarkers could be applied to diagnosis, prognosis, and management of inflammatory rheumatic diseases. Laboratory biomarkers provide an invaluable foundation to understand pathogenesis and develop new therapies, such as biological agents, and are informative in groups of patients.

Nonetheless, laboratory tests have many limitations in the care of individual patients with rheumatic diseases. For example, in patients with rheumatoid arthritis (RA), RF is positive in only $69 \%^{3}$, anti-cyclic citrullinated peptide (anti-CCP) antibodies are positive in only $67 \%{ }^{3}$, and erythrocyte sedimentation rate (ESR) is greater than $28 \mathrm{~mm} / \mathrm{h}$ in only $60 \%{ }^{4}$. Only about 1 in $50-100$ people with a positive ANA has systemic lupus erythematosus (SLE) ${ }^{5}$. Indeed, no laboratory test (or any other single measure) can serve as a "gold standard" test for diagnosis and monitoring of all individuals with any rheumatic disease ${ }^{5}$.

Recognition of the limitations of laboratory tests in rheumatology patient care has led to development of pooled indices, such as the RA Core Data Set, Disease Activity Score (DAS), and Clinical Disease Activity Index (CDAI); the SLE Disease Activity Index (SLEDAI); Bath Ankylosing Spondylitis Disease Activity Index (BASDAI); Birmingham Vasculitis Activity Score; Western Ontario and McMaster
University Osteoarthritis scale; Fibromyalgia Impact Questionnaire; and others (see Pincus and Sokka ${ }^{6}$ ). Each index includes quantitative patient self-report data to depict patient status.

These indices are widely used in clinical trials and other clinical research. However, they are generally too complex for usual care. Indeed, most rheumatology care is conducted without quantitative measures to inform clinical decisions and document the value of patient status, other than laboratory tests, the limitations of which formed the original basis for development of the clinical indices!

This situation suggests a return to the KISS principle to quantitate patient status, but with a significant departure from the traditional simple measures — patient self-report measures rather than (or in addition to) laboratory tests. Information from patients generally provides the foundation for most rheumatology clinical decisions regarding diagnosis and treatment, and is included in most indices. Why not regard patient measures, rather than laboratory tests, as the most valuable simple quantitative measures to assess and document care?

Of course, it is possible to oversimplify, such as the traditional 1-4 scales for functional class and radiographic score $^{15}$. These are robust measures, but insensitive to change. By contrast, 0-10 scales for the 3 self-report measures in the RA Core Data Set, i.e., physical function, pain, and patient estimate of global status, distinguish between active and control treatments in RA clinical trials as efficiently as the other 4 Core Data Set measures, i.e., swollen joint count, tender joint count, physician global estimate, or acute-phase reactant (ESR or C-reactive protein) ${ }^{7}$. The Health Assessment Questionnaire (HAQ) or multidimensional HAQ (MDHAQ) physical function score, not a laboratory test or radiograph, is the most significant predictor of severe outcomes of RA, such as work disability or premature mortality ${ }^{8}$, in 17 of 18 studies that have included all 3 types of measures. Pain is the primary reason that patients seek rheumatology care. Patient global estimate is the most representative measure of status.

Application of the KISS principle has led to a simple Personal non-commercial use only. The Journal of Rheumatology Copyright $\odot$ 2009. All rights reserved. 
index known as the Routine Assessment of Patient Index Data 3 (RAPID3), scored 0-30, based on the three 0-10 scores for physical function, pain, and patient global estimate. RAPID3 is correlated with DAS28 and CDAI in clinical trials ${ }^{9}$ and clinical care, and also distinguishes active from control treatments as effectively as DAS28 and CDAI. RAPID3 is scored in less than 10 seconds $^{11}$ on a one-page multidimensional $\mathrm{HAQ}$, with scoring templates, 21-circle visual analog scales, and boxes to enter the data. By contrast, scoring requires 42 seconds for a traditional HAQ, 94 seconds for a formal joint count, 106 seconds for a CDAI, and 114 seconds for a DAS28.

Patient flow is not disrupted at all if the MDHAQ is distributed to all patients by a receptionist in the infrastructure of care ${ }^{12}$, rather than in the examination room, after the visit, or selectively to certain patients. The MDHAQ provides a review of systems and recent medical history information for the physician to help manage patient care, which can save several minutes per patient. The treating physician should have the data for review with patients with all rheumatic diseases ${ }^{8}$, ideally using a flow-sheet, to inform clinical decisions in patients. Further application of the KISS principle is seen in newer software systems such as GoTreatIT (DiaGraphIT AS, Kristiansand, Norway; www.diagraphit.com), a Norwegian software management system, for efficient data recording and management.

Patient questionnaires were initially regarded as surrogates for more "objective" measures such as laboratory tests and radiographs. However, patient self-report Core Data Set measures appear to be as reliable (reproducible) as joint count measures obtained by a physician ${ }^{13}$. Their effectiveness in clinical trials, prognosis, and documentation compared to traditional joint count and radiographic and laboratory data is extensively recognized ${ }^{14}$.

An MDHAQ-RAPID3 score is useful in all rheumatic diseases, including SLE, ankylosing spondylitis, vasculitis, psoriatic arthritis, gout, osteoarthritis, fibromyalgia, and Behçet's disease ${ }^{6}$. Of course, indices such as a SLEDAI, BASDAI, or other specialized index are more comprehensive, and will always be valuable in clinical research. Formal studies to compare the feasible RAPID3 to SLEDAI, BASDAI, and other indices would be of value, as feasibility is a stated aim of the outcome measures in RA clinical trials (OMERACT) program.

Patient scores for physical function, pain and global estimate, and RAPID3 may be regarded as "vital signs" in chronic rheumatic diseases ${ }^{14}$, according to the KISS principle. Clinical decisions are based primarily upon information from a patient. RAPID3 can quantify that information to provide numbers rather than impressions for comparison from one visit to the next, with minimal cost and professional time. MDHAQ-RAPID3 might be included at all visits of all patients in the infrastructure of rheumatology care $^{12}$.
THEODORE PINCUS, MD,

New York University Hospital for Joint Diseases, New York, New York, USA;

TUULIKKI SOKKA, MD,

Jyväskylä Central Hospital, Jyväskylä, and Medcare Oy, Äänekoski, Finland

Address reprint requests to Dr. T. Pincus, NYU Hospital for Joint Diseases, 301 East 17th Street, New York, NY 10003. E-mail: tedpincus@gmail.com

\section{REFERENCES}

1. Rose HM, Ragan C, Pearce E, Lipman MO. Differential agglutination of normal and sensitized sheep erythrocytes by sera of patients with rheumatoid arthritis. Proc Soc Exp Biol Med 1948;68:1-6.

2. Hargraves MM, Richmond H, Morton R. Presentation of two bone marrow elements: The "tart" cell and "L.E." cell. Proc Staff Meet Mayo Clin 1948;23:25-8.

3. Nishimura K, Sugiyama D, Kogata Y, et al. Meta-analysis: Diagnostic accuracy of anti-cyclic citrullinated peptide antibody and rheumatoid factor for rheumatoid arthritis. Ann Intern Med 2007; 146:797-808.

4. Wolfe F, Michaud K. The clinical and research significance of the erythrocyte sedimentation rate. J Rheumatol 1994;21:1227-37.

5. Pincus T. Advantages and limitations of quantitative measures to assess rheumatoid arthritis: joint counts, radiographs, laboratory tests, and patient questionnaires. Bull Hosp Joint Dis 2006;64:32-9.

6. Pincus T, Sokka T. Can a Multi-Dimensional Health Assessment Questionnaire (MDHAQ) and Routine Assessment of Patient Index Data (RAPID) scores be informative in patients with all rheumatic diseases? Best Pract Res Clin Rheumatol 2007;21:733-53.

7. Pincus T, Amara I, Segurado OG, Bergman M, Koch GG. Relative efficiencies of physician/assessor global estimates and patient questionnaire measures are similar to or greater than joint counts to distinguish adalimumab from control treatments in rheumatoid arthritis clinical trials. J Rheumatol 2008;35:201-5.

8. Sokka T, Abelson B, Pincus T. Mortality in rheumatoid arthritis: 2008 update. Clin Exp Rheumatol 2008;26 Suppl:S35-61.

9. Pincus T, Bergman MJ, Yazici Y, Hines P, Raghupathi K, Maclean R. An index of only patient-reported outcome measures, Routine Assessment of Patient Index Data 3 (RAPID3), in two abatacept clinical trials: similar results to Disease Activity Score (DAS28) and other RAPID indices that include physician-reported measures. Rheumatology 2008;47:345-9.

10. Pincus T, Swearingen CJ, Bergman M, Yazici Y. RAPID3 (Routine Assessment of Patient Index Data 3), a rheumatoid arthritis index without formal joint counts for routine care: Proposed severity categories compared to Disease Activity Score and Clinical Disease Activity Index categories. J Rheumatol 2008;35:2136-47.

11. Yazici Y, Bergman M, Pincus T. Time to score quantitative rheumatoid arthritis measures: 28-joint count, Disease Activity Score, Health Assessment Questionnaire (HAQ), multidimensional HAQ (MDHAQ), and Routine Assessment of Patient Index Data (RAPID) scores. J Rheumatol 2008;35:603-9.

12. Pincus T, Wolfe F. An infrastructure of patient questionnaires at each rheumatology visit: Improving efficiency and documenting care. J Rheumatol 2000;27:2727-30,

13. Kvien TK, Mowinckel P, Heiberg T, et al. Performance of health status measures with a pen based personal digital assistant. Ann Rheum Dis 2005;64:1480-4.

14. Pincus T. Pain, function, and RAPID scores - Vital signs in chronic diseases, analogous to pulse and temperature in acute diseases and blood pressure and cholesterol in long-term health. Bull NYU Hosp Jt Dis 2008;66:155-65. 